\title{
AGENTES PÚBLICOS DE LA RECREACIÓN EN URUGUAY
}

\author{
PUBLIC AGENTS OF RECREATION IN URUGUAY
}

AGENTES PÚBLICOS DA RECREAÇÃO NO URUGUAI

\section{Resumen}

El artículo plantea los principales hallazgos de un estudio exploratorio realizado durante el 2017, donde se relevó y analizó la presentación de 41 programas recreativos o con componente recreativo, llevados adelante por diversos agentes públicos del Estado en Uruguay. El objetivo fue identificar la presencia de la recreación en la ejecución de políticas públicas por parte de la actual gestión de gobierno en Uruguay (2015-2019), a partir de la presentación que hacen los propios actores en las páginas web oficiales. Los hallazgos del estudio dan cuenta de una gran dispersión de los programas recreativos y la ausencia de un agente rector de las políticas de recreación, además de una fuerte tendencia hacia el recreacionismo.

Palabras clave: recreación; administración pública; programas recreativos; Uruguay

\section{Abstract}

The article posits the main findings of an exploratory research carried out during 2017, in which the authors collected and analyzed the presentation of 41 recreational programs or programs with a recreational component, run by diverse State public agents in Uruguay. The aim was to identify the presence of recreation policies in the execution of public policies by the current government administration in Uruguay (2015-2019). The findings of the study show a great dispersion of recreational programs and the absence of a guiding agent of recreation policies, as well as a strong tendency towards recreationism.

Keywords: recreation; public administration; recreational programs; Uruguay

1 Doctor en Ocio y Desarrollo Humano; y licenciado en Comunicación Social con posgrado en Educación en Valores. Universidad Católica del Uruguay. Correo electrónico: rlema@ucu.edu.uy.

2 Magíster en Estudios Internacionales de Ocio. Profesor de Educación Física. Universidad Católica del Uruguay. Correo electrónico: gustavo.martinez@ucu.edu.uy. 


\section{Resumo}

0 artigo apresenta as principais conclusões de um estudo exploratório realizado durante 2017, onde foi revelada e analisada a apresentação de 41 programas recreativos ou com componentes recreativos, realizados por diversos agentes públicos do Estado do Uruguai. 0 objetivo foi identificar a presença da recreação na execução de políticas públicas pelo atual governo do Uruguai (2015-2019), com base na apresentação feita pelos próprios atores nos sites oficiais. As conclusões do estudo mostram uma grande dispersão de programas recreativos e a ausência de um agente governante de políticas de recreação, além de uma forte tendência para a recreação.

Palavras chave: recreação; administração pública; programas recreativos; Uruguai

Fecha de recepción: 1 de septiembre de 2016

Fecha de aprobación: 11 de septiembre de 2018

\section{Para citar este artículo}

Lema, R. y Martínez, G. (2018). Agentes públicos de la recreación en Uruguay. Lúdica Pedagógica, 27, x-xx. 
Actualmente la recreación organizada en Uruguay se refiere a un conjunto de prácticas diversas, que se dan en una gran variedad de ámbitos e instituciones públicas y privadas. Si bien en muchos espacios suele ser una práctica asociada a la educación física, lo que implica priorizar juegos motrices y deportivos que permiten dar a la población diversas opciones de disfrute, cada vez son más las propuestas que se asocian con prácticas educativas con componentes lúdicos y recreativos que van más allá de lo motriz y configuran un campo de acción e intervención propio.

En Uruguay el Estado asume un rol explícito en el ámbito de la recreación a partir de 1911, con la creación de la Comisión Nacional de Educación Física, organismo rector de las políticas de educación física, deporte y recreación a lo largo del siglo $\mathrm{xx}^{3}$. Como estrategia principal se destaca desde el comienzo el desarrollo de las Plazas Vecinales de Cultura Física, luego denominadas Plazas de Deporte, un modelo basado en los playgrounds y parques estadounidenses, que en sus primeros años se definirá como un centro recreativo para el desarrollo de actividades físicas, lúdicas y deportivas (Lema, 2015). Sin embargo, la hegemonía de este agente ya no es tan evidente, y han surgido otros actores en el ámbito público que desempeñan un papel relevante en el campo de la recreación.

\section{MARCO TEÓRICO}

Hoy en Uruguay y en varios países del continente podemos distinguir diversas formas de intervención recreativa, algunas de las cuales ponen más énfasis en el consumo de actividades en tanto que otras ponen el foco en la vivencia de la experiencia recreativa. Las primeras se suelen llamar recreacionistas, por cuanto son herederas de la tradición anglosajona, mientras que las segundas se corresponden con lo que definiremos como recreación educativa. Debido a que el recreacionismo tiene su raíz en el higienismo anglosajón de principios del siglo xx y está estrechamente vinculado a la tradición de la educación física, siendo los juegos motrices y la actividad física deportiva sus instrumentos más visibles, el enfoque sociocultu-

3 Con la creación del Ministerio de Deporte y Juventud en 1995 y luego Ministerio de Turismo y Deporte, la antigua CNEF se transforma en la Dirección Nacional de Deporte (Dinade). En el actual periodo de gobierno esta entidad se transforma en la Secretaría Nacional de Deporte (SND), con rango ministerial. ral y más adelante el educativo darán cuenta de una relectura pedagógica que se da básicamente en Latinoamérica, y que incorpora prácticas de expresión y creación y otro tipo de juegos (Lema, 1999; Waichman, 2004). Hoy la recreación en nuestro país ha evolucionado del tradicional enfoque recreacionista de la Comisión Nacional de Educación Física (CNEF) hacia prácticas más cercanas a lo sociocultural y educativo, sin embargo, el Estado no siempre refleja este cambio de enfoque.

Sintetizando los aportes de diversos modelos de recreación educativa, Lema (2015) define la recreación educativa como:

[...] una práctica pedagógica que interviene en las posibilidades de disfrute del tiempo libre en un grupo o comunidad, movilizando la capacidad lúdica del sujeto para recrear su entorno, creando así mejores condiciones para la participación de la comunidad en su propio desarrollo humano, en un paulatino proceso hacia la construcción de la autonomía de todos los sujetos implicados. (p. 82).

La evolución de la recreación hacia un enfoque educativo será acompañada por el surgimiento de una gran variedad de agentes institucionales que desarrollarán diversos programas recreativos. Ander-Egg (1974) define al agente de una intervención como aquel componente institucional o personal que lleva adelante una acción socioeducativa y que tiene la capacidad de producir algún efecto en el campo. Desde el punto de vista de la recreación educativa, el agente es una organización que adelanta la acción socioeducativa, con capacidad para generar resultados de aprendizaje en el desarrollo humano (Lema, 2012).

En el caso de Uruguay, el Estado es el principal agente político que regula las intervenciones y financia muchas de las propuestas. Si bien durante buena parte del siglo xx estas se concentraban en un único agente (la CNEF), hoy la recreación está dispersa en diversas instituciones nacionales y regionales.

Nos interesa en este estudio analizar la dimensión de praxis política en recreación, aquella que refiere a la forma en que estas políticas se instrumentan a través de un conjunto de agentes y acciones. Según San Salvador del Valle (1996) la práctica política tiene un marco de referencia, adopta una estructura y se desarrolla a través de acciones. En este sentido nos interesará indagar principalmente acerca de las 
estructuras que asumen el rol de agentes recreativos en el ámbito público y la organización de las acciones recreativas a través de programas.

El Estado interviene como un actor más en el campo de la recreación y los procesos de intervención política en su caso implican la regulación normativa, la planificación, la programación y la gestión de los recursos (San Salvador del Valle, 1996). Si bien no es suficiente la existencia de acciones y estructuras para poder hablar de políticas de recreación, estas son condición necesaria. "La consideración y la denominación de una intervención como políticas exige, como primera condición, un número suficiente de acciones generadas para tal fin" (p. 262). Por ello es necesario conocer los agentes y programas recreativos que existen en el ámbito público, para poder evaluar la existencia y calidad de una política específica.

Hoy las políticas de recreación no se pueden entender por fuera de los derechos de los ciudadanos al disfrute de su tiempo libre. Esto crea obligaciones, especialmente al Estado, que debe ser garante de ese derecho; por ello la política pública debe apuntar a restituir el derecho a la recreación, en particular de los sectores más vulnerables. Como plantea Contreras (2005), desde un enfoque de derechos el Estado no solo debe garantizar ese derecho, facilitando el acceso a los bienes de ocio, sino que también debe facilitar la participación de los beneficiarios en la definición y ejecución de las políticas, además de rendirles cuentas de las acciones que emprende para concretar esas estrategias.

La acción política del Estado se instrumenta a través de programas que pueden ser específicos de la recreación o tomar elementos recreativos para el desarrollo de fines extrínsecos. En este sentido, entendemos que un programa recreativo reúne aquellas propuestas que de alguna manera explicitan en sus objetivos la recreación y el tiempo libre, y por extensión la cultura, el deporte y el turismo. Pero muchas veces encontramos programas que, a pesar de no ser específicamente recreativos, incorporan propuestas recreativas como parte de su estrategia. A estos últimos los consideraremos programas con componente recreativo, ya que, si bien incorporan la recreación como parte sustancial de su estrategia, tienen una finalidad extrínseca a la recreación.
En este trabajo se diferencian los programas recreativos o con componente recreativo, a partir de los objetivos que explicitan los casos relevados. Para el análisis de los objetivos, a su vez, se toma en cuenta el tipo de enfoque del programa, los beneficios a los que apunta y las dimensiones de desarrollo que contempla. En cuanto al enfoque del programa, distinguiremos aquellos que están centrados en una oferta de actividades de aquellos que apuntan al desarrollo de una vivencia de largo plazo. Según Cuenca (2004) los primeros se centran en una oferta de actividades que el beneficiario elige para su consumo, en tanto que los segundos se centran en la vivencia de una experiencia satisfactoria en el marco de un proceso de intervención.

Como segundo criterio para analizar los objetivos se considera la clásica distinción de Driver y Burns (1999) sobre los tipos de beneficios: aquellos que implican la mejora de una condición o situación de la persona, grupo o entidad; aquellos que procuran la prevención de una condición no deseada o el mantenimiento de una condición deseada; y por último, aquellos que apuntan a la consecución de una experiencia de ocio satisfactoria.

Por último, para analizar la dimensión del desarrollo que se prioriza en los objetivos, se toman en cuenta las dimensiones propuestas por Lema y Machado (2013): programas que apuntan al desarrollo cultural del individuo y su comunidad, programas que apuntan al fortalecimiento social o a la mejora de las relaciones de convivencia y programas que apuntan al desarrollo y crecimiento personal.

Otro aspecto por analizar será el tipo de población al que apuntan los programas recreativos, considerando la segmentación etaria y destacando algún otro elemento de segmentación que dé cuenta de la atención a alguna circunstancia en particular, como el caso de situaciones de vulnerabilidad (discapacidad, pobreza, necesidades básicas insatisfechas, etc.).

Por último, se analiza el tipo de actividades recreativas que llevan adelante estos programas. Para ello se toma la tipología de Lema y Machado (2013), que distinguen entre actividades lúdicas o juegos, físico-deportivas, al aire libre, de expresión y creatividad, de difusión y consumo cultural, de turismo recreativo, festivas, solidarias y de formación desinteresada o voluntariado. 
El propósito de este análisis será visualizar la presencia de la recreación en el accionar de diversos agentes públicos del Estado nacional, procurando identificar el papel que desempeña hoy el Estado uruguayo en el desarrollo y la ejecución de una política de recreación.

\section{DISEÑO DE INVESTIGACIÓN}

En cuanto al alcance de esta investigación, este es un estudio exploratorio y de corte cualitativo. La metodología elegida fue el análisis documental de las páginas web oficiales de diferentes dependencias estatales, en el entendido de que allí cada agente hace explícita su razón de ser y hace públicas las propias representaciones sobre las prácticas que adelantan.

El diseño de la investigación estará guiado por dos preguntas principales: ¿Qué agentes públicos operan actualmente en el campo de la recreación? Y ¿Existe en la actualidad un ente rector de las políticas de recreación en el Uruguay? El principal objetivo de investigación será identificar la presencia de la recreación en la ejecución de políticas públicas por parte de la actual gestión de gobierno en Uruguay (20152019), considerando la presentación que hacen los propios actores estatales en sus páginas web oficiales.

El relevamiento se realizó entre mayo y junio del 2017, cumplidos dos años de la actual gestión presidencial, suponiendo que para entonces ya estaban instrumentados los posibles cambios de políticas respecto al anterior periodo de gobierno. Para este relevamiento se tuvieron en cuenta un conjunto de variables ya presentadas en el marco teórico y que se retomarán en el análisis.

Como universo de la investigación se toman en cuenta todas las instituciones públicas del primer nivel de gobierno (Estado nacional). Esto incluye a la Administración central, a organismos descentralizados y autónomos. No se consideran en este momento de la investigación las instituciones de gobierno departamental y municipal.

El proceso de investigación tuvo un momento de relevamiento que implicó la revisión de páginas webs oficiales de los diferentes agentes públicos, analizando los diferentes programas e identificando posibles intervenciones o actividades recreativas. En los casos en que esta identificación no era tan evidente, se pro- cedió a utilizar los motores de búsqueda de la propia página, escribiendo como palabra clave recrea, ya que esta contempla las diferentes desambiguaciones del término recreación. A partir de esta búsqueda se identificaron 18 agentes que desarrollan programas recreativos o con componente recreativo, en los que se centró el análisis (véase el cuadro 1).

Una vez identificados los programas, se procedió a relevar los siguientes datos: nombre del organismo y dependencia, nombre del programa, objetivos del programa, público al que se dirige y actividades desarrolladas. Luego de completar el relevamiento, se procesaron los datos en función de las variables de análisis presentadas en el marco teórico. A continuación se presentan los hallazgos correspondientes.

Cuadro 1. Agentes relevados

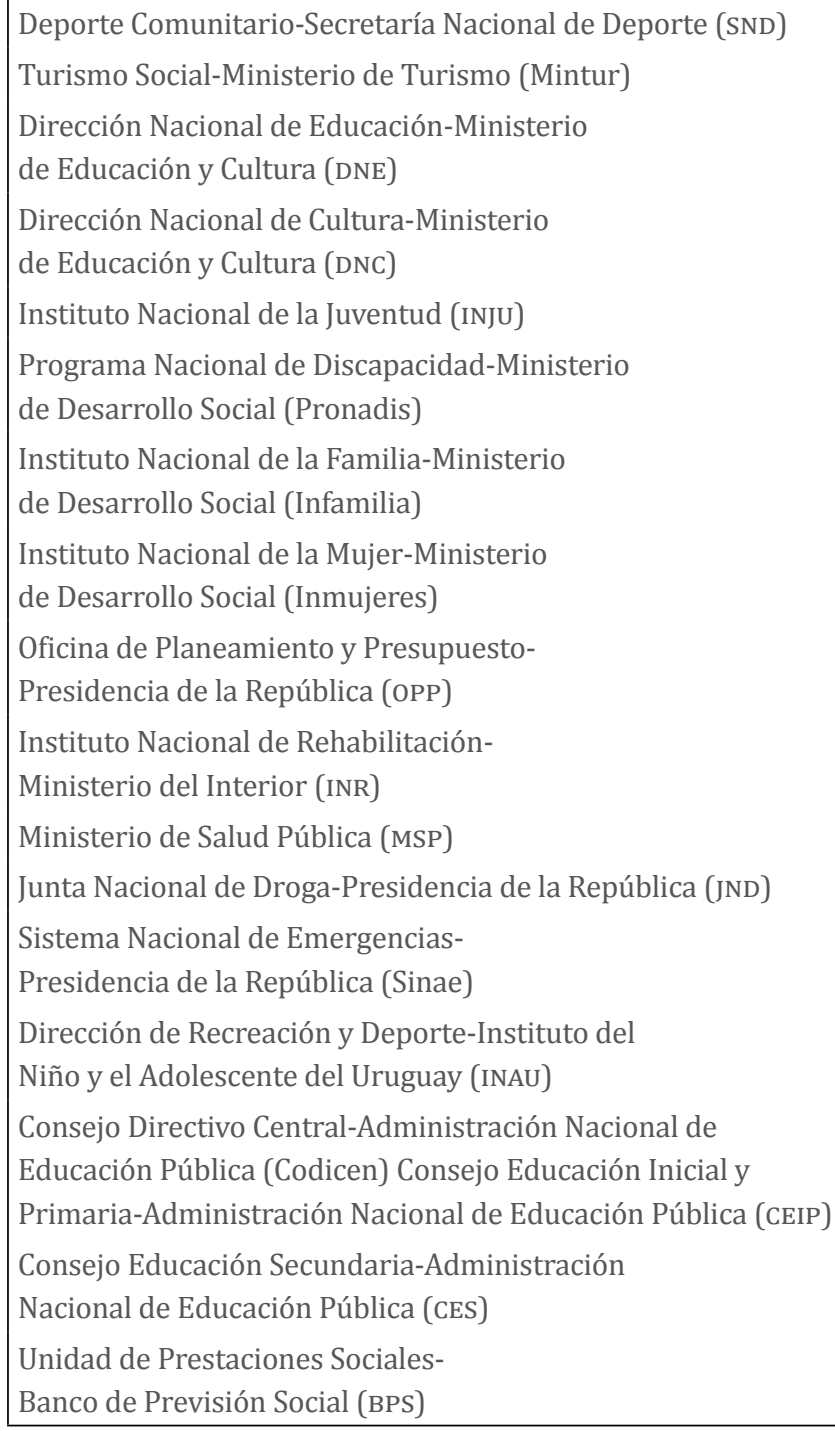

Fuente: elaboración propia. 


\section{DISCUSIÓN DE LOS HALLAZGOS}

Del relevamiento surge que hay al menos 41 programas que se desarrollan a través de acciones recreativas, estructurados en un total de 18 agentes institucionales. A diferencia de lo que sucedía en el pasado, cuando la CNEF, hoy Secretaría Nacional de Deporte, era identificada como el organismo rector de las políticas de recreación, hoy nos encontramos con que este agente no cuenta con una unidad específica para el desarrollo de planes y programas recreativos. Y al tiempo que la recreación ha desaparecido de la estructura de la SND, se pueden identificar unidades de recreación en otros organismos estatales sectoriales, como en el Programa Nacional de Discapacidad (área de Cultura, Deporte y Recreación), en el Instituto Nacional de Rehabilitación (Coordinación de Deporte y Recreación), y en el Instituto del Niño y el Adolescente del Uruguay (Departamento de Recreación Educativa y Deporte).

Como decíamos, se identificaron en total 41 programas de recreación. De estos, 16 son explícitamente recreativos de acuerdo con sus objetivos, aunque también otros 8 podrían considerarse recreativos por el tipo de actividades que desarrollan y por su énfasis en el beneficio intrínseco. Por lo tanto podemos considerar que 24 programas son específicamente recreativos, en tanto que los otros 17 cuentan con un componente recreativo.

Analizando los ocho programas que hemos considerado recreativos, pese a no ser explícitos, encontramos que cinco de estos son claramente identificables con la recreación y que se ejecutan a través de actividades propias del campo de la recreación. Estos son:

- Cultura y Recreación (área Turismo Social, del Banco de Previsión Social)

- Programa de Actividades Recreativas y Expresivas de las Escuelas de Tiempo Completo (Consejo de Educación Inicial y Primaria)

- Programa de Tiempo Extendido (Consejo de Educación Inicial y Primaria)

- Programa Educativo de Verano (Consejo de Educación Inicial y Primaria)

- Talleres recreativos de Liceos de Tiempo Extendido y Tiempo Completo (Consejo de Educación Secundaria)
Llama la atención la ausencia de un reconocimiento de la especificidad recreativa en estos casos y un intento por justificarse en fines con mayor legitimidad social. Es así que estos programas buscan definirse con fines educativos ("extensión del tiempo pedagógico", "educación integral"), sociales ("desarrollo de habilidades para la convivencia") e incluso de salud ("promocionar hábitos saludables y generar valores", "mantenimiento de la salud").

Lo mismo sucede con otros tres programas que, si bien se enfocan en el desarrollo de experiencias satisfactorias, no se reconocen como explícitamente recreativos:

- ProArte (Consejo Directivo Central, Administración Nacional de Enseñanza Pública)

- Encuentros de Arte y Juventud (Instituto Nacional de la Juventud)

- Casa Joven (Instituto Nacional de la Juventud)

Por último, se identifican 17 programas que, aunque no son propiamente recreativos, incorporan un componente recreativo en su intervención, lo que quiere decir que la recreación en estos casos es un instrumento para fines extrínsecos.

En cuanto al análisis de los dieciocho agentes institucionales identificados en el relevamiento, podemos ver que once de estos desarrollan programas específicamente recreativos, en tanto que el resto solo desarrolla programas con componente recreativo. Entre los primeros se destacan el Banco de Previsión Social y el Consejo de Educación Inicial y Primaria, con cuatro programas cada uno. Luego le siguen la Secretaría Nacional del Deporte y el Instituto del Niño y el Adolescente del Uruguay, con tres programas cada uno; la Dirección Nacional de Cultura y el Consejo Directivo Central de la Administración Nacional de Educación Pública, con dos programas; y los cinco restantes con un programa cada uno.

Se evidencia así una gran dispersión de los programas recreativos, siendo varios los agentes públicos que ocupan un lugar destacado en el campo de la recreación y sin que ninguno de ellos sobresalga. De estos datos se puede inferir que, a diferencia de lo que sucedió durante el siglo $\mathrm{xx}$, no hay actualmente un agente rector de la recreación en el Uruguay. 


\section{Objetivos de la recreación}

De los 41 programas relevados, 24 apuntan a desarrollar una oferta de actividades a disposición del público objetivo. Los 17 restantes se dirigen al desarrollo de un proceso vivencial de largo plazo, donde la recreación es un componente destacado de la intervención.

Entre los agentes que se orientan mayormente a la oferta de actividades, el Banco de Previsión Social (BPS), la Dirección Nacional de Cultura (DNC), el Instituto del Niño y el Adolescente del Uruguay (INAU) y la Secretaría Nacional de Deporte (SND) son los que más programas desarrollan. Los programas del Consejo Directivo Central (Administración Nacional de Enseñanza Pública-ANEP) y del Instituto Nacional de la Juventud (INJU) apuestan, en cambio, al desarrollo de procesos vivenciales en los cuales el componente recreativo es un aspecto clave de la intervención educativa o sociocultural, respectivamente.

En cuanto a los beneficios que promueven estos programas, podemos identificar 15 de ellos que apuntan al beneficio intrínseco (consecución de una experiencia satisfactoria). En esta categoría se destaca el rol que desempeñan algunos agentes como el BPS, los diversos Consejos de Educación de la ANEP, el INAU, la DNC y el Ministerio de Turismo.

Otros quince programas, en su mayoría con componente recreativo, apuntan a la prevención de situaciones de riesgo personal o social, siendo diversos los agentes involucrados en esta categoría. Y nueve programas del INJU y otros agentes apuntan a la mejora de una condición social y al desarrollo cultural de la población. En dos casos no fue posible determinar qué tipo de beneficio buscan los programas.

Repasando únicamente los 24 programas que hemos considerado como propiamente recreativos, encontramos que la mayoría (15) están orientados al beneficio intrínseco y con mayor énfasis en el desarrollo de la dimensión social (13). Por otra parte, la mayoría de estos programas están centrados en la oferta de actividades recreativas (17), lo que da cuenta de un predominio del enfoque recreacionista en el ámbito público.

En cuanto a los programas con componente recreativo, recordamos que estos asumen la recreación como un instrumento para fines extrínsecos. Tomando en cuenta los beneficios, 10 de los 17 programas la utilizan para prevenir situaciones personales y sociales, y 7 de ellos para mejorar las condiciones sociales y el desarrollo cultural.

\section{Públicos a los que se dirigen estos programas}

Si bien los datos que surgen del relevamiento en cuanto a la población a la que se dirigen los diferentes programas son muy básicos y no dan cuenta de la cobertura de diferentes tipos de públicos, igualmente se puede percibir que hay propuestas diversas y equilibradas respecto a los segmentos de edad.

De los datos obtenidos surge que hay propuestas específicas para atender diversas situaciones de vulnerabilidad (privación de libertad, situación de calle, bajos ingresos) y necesidades insatisfechas (comunidades locales con escaso acceso a bienes culturales), pero sin dar cuenta del volumen de cobertura o población atendida. Solo hay un programa dirigido a público en situación de discapacidad.

\section{Tipo de actividades que}

\section{desarrollan los programas}

En cuanto al análisis de las actividades recreativas que se llevan adelante en el total de programas relevados, vemos un claro predominio de las actividades con énfasis lúdico. Es así que 18 programas desarrollan actividades de expresión y creación, y otros 17 desarrollan actividades de juego. También se destacan las actividades físico-deportivas y las que se practican al aire libre, en 13 y 10 programas respectivamente. En menor medida las actividades de turismo recreativo (6 programas), las de consumo cultural (5), las solidarias (3) y las festivas (1) (véase la gráfica 1).

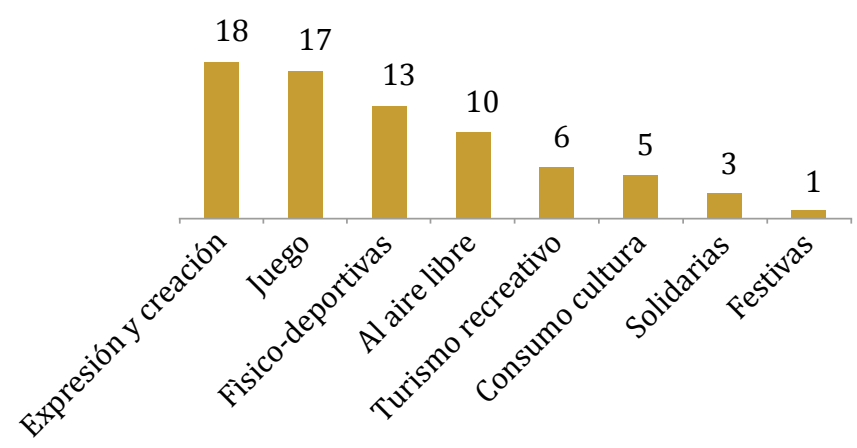

Gráfica 1. Tipo de actividades en programas recreativos y con componente recreativo.

Fuente: elaboración propia. 
Un primer análisis nos permite destacar un énfasis lúdico en las actividades desarrolladas por este programa. Predominan los juegos en general y otras actividades vinculadas a la expresión y creación, ambas más vinculadas con un enfoque educativo de la recreación. Sin embargo, cuando tomamos en cuenta solo a los 24 programas propiamente recreativos, este predominio se modifica. En el caso de los programas recreativos, prevalecen las actividades lúdicas y al aire libre ( 9 programas en cada caso) y en segundo lugar las físico-deportivas y las de expresión y creación (7 programas), seguidas de cerca por las de turismo recreativo (6), en tanto que las de consumo cultural (4) y las festivas (1) siguen siendo minoritarias (véase la gráfica 2 ).

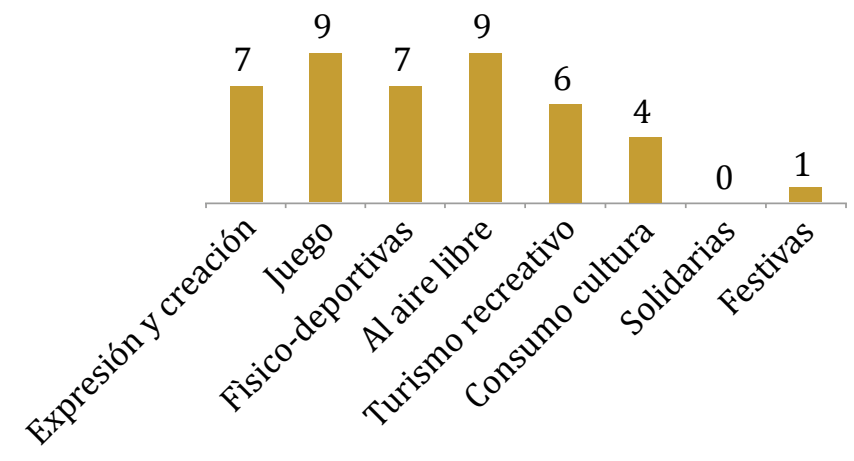

Gráfica 2. Tipo de actividades en programas específicamente recreativos

Fuente: elaboración propia.

En este caso la presencia de actividades físico-deportivas y al aire libre, algo que se suele identificar con el enfoque recreacionista, se equipara con las referencias a actividades de expresión y creación y a los juegos en general. En este sentido nos preguntamos por qué los programas recreativos en el ámbito público desarrollan actividades más vinculadas con el recreacionismo, en tanto que los programas no específicos que incorporan un componente recreativo desarrollan otro tipo de actividades más identificadas con un enfoque educativo. Probablemente esta situación dé cuenta de la influencia que la educación física mantiene en aquellos programas que se reconocen a sí mismos como recreativos, al menos en el ámbito público. Así, se puede inferir que los programas específicamente recreativos siguen con un vínculo fuerte en la tradición de la educación física, mientras que otras disciplinas desempeñan un papel más destacado en aquellos programas que no son propiamente recreativos, lo que aporta a la diversidad de propuestas.

\section{CONCLUSIONES}

El estudio ha permitido un primer acercamiento a las políticas de recreación que se desarrollan en la actualidad en el ámbito público, especialmente en el primer nivel de gobierno. De los datos relevados se pudo identificar 18 agentes que se desempeñan en el campo de la recreación a través de 41 programas recreativos o con componente recreativo. Se constata así la existencia de un importante número de programas, pero también su dispersión en diversos agentes. No identificamos una política de recreación articulada e integrada en un único agente institucional. Por lo tanto no podemos hablar hoy de un órgano rector de estas políticas, al perder la Secretaría Nacional de Deporte el protagonismo que ejerció su antecesora (CNEF) a lo largo del siglo xx en el campo de la recreación.

Otro elemento que destacamos entre los hallazgos es la dificultad que tienen los agentes públicos para reconocer algunos de sus programas como explícitamente recreativos. Si bien excede el alcance de esta etapa de investigación, una subvaloración de lo recreativo podría explicar la dificultad por parte de algunos organismos del Estado para reconocerse como agentes de la recreación. Los intentos de justificar la acción recreativa en fines educativos, sociales o de salud dan cuenta de debilidades en la legitimidad social de una política orientada a fines específicos de recreación y disfrute del tiempo libre.

La mayoría de los programas específicamente recreativos están orientados a la oferta de actividades y con un mayor protagonismo de juegos motores y actividades físico-deportivas, lo que demuestra un mayor predominio del recreacionismo en estos agentes del ámbito público. Sin embargo, los que desarrollan programas con componente recreativo están más abiertos a otro tipo de actividades (expresivas y juegos en general) y al desarrollo de un proceso vivencial, elementos que dan cuenta de una tendencia hacia el enfoque educativo que enriquece el ámbito público de la recreación. En este sentido, creemos importante considerar el papel de estos actores a la hora de construir un campo recreativo de mayor complejidad y con mayor autonomía respecto a la educación física. 
El estudio es un primer intento de conocer y analizar un conjunto de experiencias en el ámbito de la política pública, cuyo alcance se vio limitado al no considerar en esta oportunidad los niveles departamental y municipal de gobierno, donde sin duda hay numerosos agentes públicos que aportan e intervienen en el campo de la recreación. A su vez, la falta de acceso a otras fuentes documentales restringió las posibilidades de un análisis con mayor profundidad, que tuviera en cuenta la realidad de la ejecución y de los resultados alcanzados por dichos programas. Igualmente creemos que este estudio puede aportar al conocimiento de una realidad poco estudiada en nuestro país y a comparar experiencias con otros países de la región. Además, representa una oportunidad de orientar la toma de decisiones de quienes hoy tienen la responsabilidad de ejecutar las políticas públicas de nuestro campo.

Como continuidad de este estudio, consideramos pertinente profundizar algunos de los elementos reseñados. En este sentido, creemos importante indagar en las representaciones de la recreación que tienen quienes coordinan los programas analizados y conocer qué perfiles profesionales trabajan en estos. Por otra parte, es pertinente profundizar en el potencial de estos programas para el fortalecimiento de una política pública de recreación y su articulación en un plan nacional que permita asegurar el rol del Estado como garante del derecho al disfrute del tiempo libre.

\section{REFERENCIAS}

Ander-Egg, E. (1974). Diccionario de trabajo social. Buenos Aires: ECRO-ILPH.

Contreras Rodríguez, L. A. (2005). La formulación de la política pública para el deporte y la recreación del municipio de Medellín desde el enfoque de los derechos humanos. En J. F. Tabares Fernández, A. F. Ossa Montoya y V. Molina Bedoya (coords.), El ocio, el tiempo libre y la recreación en América Latina: problematizaciones y desafíos (pp. 15-46). Medellín: Civitas.

Cuenca Cabeza, M. (2004). Pedagogía del ocio, modelos y propuestas. Bilbao: Universidad de Deusto.

Driver, B. L. y Burns, D. H. (1999). Concepts and uses of the benefits approach to leisure, en E. L. Jackson y T. L. Burton (eds.). Leisure studies: Prospects for the 21st century (pp. 349-369). State College, PA: Venture.

Lema, R. (1999). Recreación, tiempo libre y educación en el Uruguay. Prisma, 11, 136-142.

Lema, R. (2012). La recreación educativa: modelos, agentes y ámbitos. RecVirtual, Revista Latinoamericana de Recreación, 1(1), 77-90.

Lema, R. (2015). La recreación educativa como trayecto de formación. Un análisis de prácticas de recreación organizada en instituciones educativas del Uruguay (tesis de doctorado inédita). Bilbao: Universidad de Deusto.

Lema, R. y Machado, L. (2013): La recreación y el juego en la intervención educativa. Montevideo: IUACJ.

San Salvador del Valle, R. (1996). Políticas de ocio. Cultura, turismo, deporte y recreación. Bilbao: Universidad de Deusto.

Tabares, J. F. (2005). El ocio y la recreación en América Latina; una lectura desde los modelos de desarrollo. En J. F. Tabares Fernández, A. F. Ossa Montoya y V. Molina Bedoya (coords.), El ocio, el tiempo libre y la recreación en América Latina: problematizaciones y desafíos (99114). Medellín: Civitas.

Waichman, P. (2004). Tiempo libre y educación, un desafío pedagógico. Buenos Aires: PW. 